\title{
L’autarchia delle acque. Mario Bettini e il moto perpetuo
}

\author{
Francesco Luzzini \\ Università degli Studi di Milano - Dipartimento di Bioscienze \\ francesco_luzzini@yahoo.com
}

Fra gli storici della scienza nessun ordine religioso ha mai suscitato più controversie e interesse della Compagnia di Gesù, fondata nel 1539 da Ignazio di Loyola (1491-1556): un valoroso capitano dell'Imperatore Carlo V che, rimasto gravemente ferito nella battaglia di Pamplona del 1521, abbandonò la spada e si dedicò con ardore alla vita consacrata.

Conversione a parte, lo spirito guerriero non lasciò mai del tutto Ignazio. Della milizia, i suoi seguaci presero molto: una ferrea disciplina, una totale devozione alla causa (quella della Controriforma) e una combattività che, nel corso della loro travagliata storia, li rese protagonisti della scena europea e, in non pochi casi, mondiale. Acerrimi difensori del cattolicesimo, i gesuiti si dedicarono a diffondere ovunque la vera religione, intraprendendo missioni in Asia, in Africa e nelle Americhe. Con altrettanto zelo arginarono la diffusione del protestantesimo in Europa: e in ciò furono implacabili, contrastando l'eresia in ogni sua forma e istituendo una rete capillare di scuole - i cosiddetti collegi - in cui formare le nuove leve dell'ordine. Ma a differenza di quanto avvenne in altre organizzazioni cattoliche, l'educazione impartita nei collegi gesuitici non si limitò alla teologia, alla filosofia e alla retorica. Essi fecero dell'insegnamento tecnico-scientifico una parte integrante e distintiva dei loro curricula, ritenendo che solo la conoscenza del sapere umano in ogni sua espressione avrebbe permesso di difendere appieno la dottrina della Chiesa. Per i gesuiti, dunque, la scienza non rappresentò mai un nemico da combattere, ma un nuovo campo di battaglia su cui confrontarsi col vero nemico, cioè ogni potenziale eresia. E se da un lato ciò li rese tra i più agguerriti oppositori delle teorie copernicane e galileiane, dall'altro li spinse a «elaborare senza complessi nuove concezioni nel campo della filosofia naturale» (Maffioli 2010), attraverso un'impressionante quantità di ricerche nei campi della matematica, dell'astronomia, dell'idraulica, e di molte altre discipline (Generali 1996).

Che si sia laici o credenti, è difficile non subire il fascino di questi lavori, frutto di un confronto coraggioso tra una fede incandescente e una preparazione tecnico-scientifica di prim'ordine. Tra il XVI secolo e la seconda metà del XVIII, molti membri della Compagnia contribuirono significativamente all'avanzamento del dibattito filosofico e scientifico moderno. E fra questi può a buon diritto annoverarsi il bolognese Mario Bettini (1584-1657), filosofo, matematico, fisico, astronomo e docente presso il collegio di Parma (Baldwin 2003; Remmert 2006).

Nel pensiero di Bettini traspaiono alcune contraddizioni tipiche di non pochi studiosi del suo tempo. Il suo enciclopedico trattato, l'Apiaria universae philosophiae mathematicae (Bettini 1642), privilegia dichiaratamente il valore teorico della matematica, intesa come la sola disciplina che, nella sua astrazione, è in grado d'avvicinare l'intelletto alla teologia (Aricò 2002); mentre la ricerca affidata ai sensi e ai prodotti della tecnica (come gli strumenti ottici) è giudicata troppo legata ai limiti umani e, dunque, poco affidabile. Eppure l'autore è un abile e rinomato astronomo, e l'impronta sperimentale è evidente nella sua opera (Baldwin 2003). Né manca l'influenza di quella filosofia neoplatonica di cui un altro gesuita, Athanasius Kircher (1602-1680), diverrà di lì a poco uno dei massimi epigoni (Luzzini 2013). Come altri suoi confratelli,

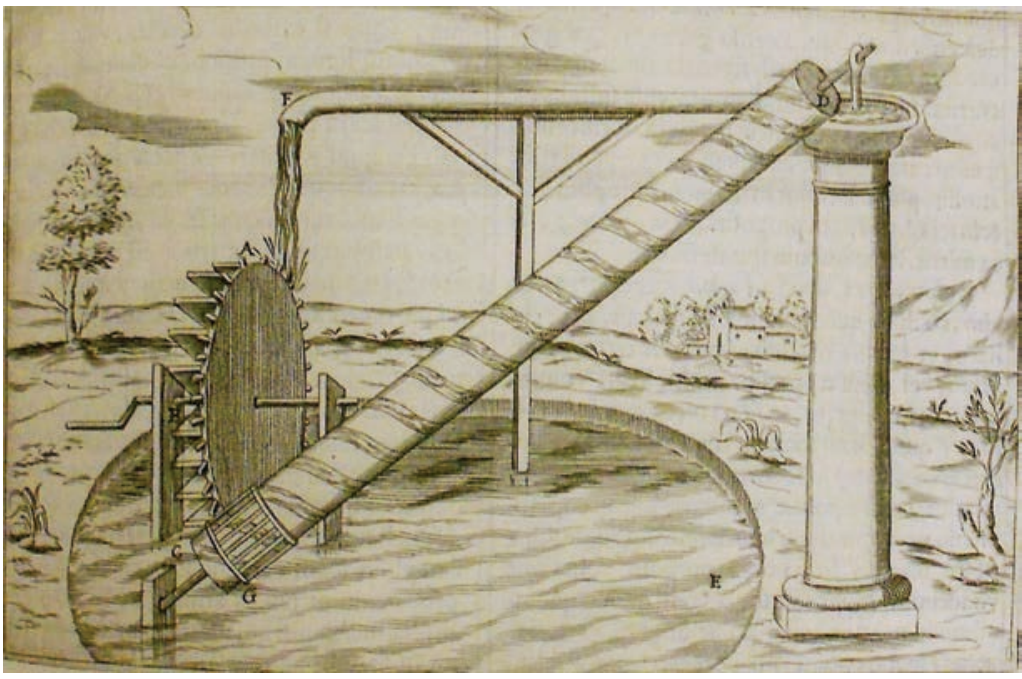

Fig. 1 - Il modello utilizzato da Bettini per descrivere il moto perpetuo delle acque. 
Bettini s'interessa al dibattito galileiano, difendendo senza esitazione il sistema geocentrico. E i suoi calcoli lo portano a elaborare diverse teorie singolari, ma rigorosamente coerenti con la pur errata premessa. Fra queste, una in particolare fa al caso nostro, giacché sostiene l'esistenza di un vero e proprio moto perpetuo delle acque («ratio motus perpetui fluviorum in orbe terrarum») con cui risolvere l'annosa questione dell'origine delle sorgenti.

Per comprendere la teoria di Bettini occorre prestare attenzione al principio cardine del sistema tolemaico, per cui la Terra è il centro dell'intero universo e, di conseguenza, anche il suo centro di gravità. La distribuzione delle acque e dei continenti non è però uniforme, ma estremamente irregolare: e ciò provoca uno sfasamento tra il centro di gravità vero e proprio e il baricentro del pianeta, o "centro di mole", come lo chiama l'autore («centrum molis, et centrum gravitatis in terrae globo non sunt in eodem puncto, quia moles ex aquis, ac terris constans magis gravitat ex ea parte, ubi terrae sunt, circa quas minores marium sinus funduntur»). La diversa distribuzione e la diversa altitudine delle terre emerse fanno sì che certe zone siano più lontane di altre dal centro di gravità dell'universo, quindi meno soggette alla stessa attrazione gravitazionale («dum igitur orbis terrarum centrum gravitatis collocatum continet in centrum universi, partes terreni globi, circa quas maria ampliora affunduntur, sunt remotiore a centrum universi, ac proinde altiores, quam reliquae partes ambitus terrestris exiguis aquis affusi»). È proprio questa condizione a innescare il movimento dell'acqua, che non è fissa come la terraferma, e dunque tende continuamente a ristabilire l'equilibrio («affectat aequilibrium»): penetra nelle cavità dei monti («per occultos terrae meatus»), risale per canali nascosti fin sulle cime, sgorga in sorgenti e forma i fiumi, che a loro volta ritornano al mare («donec in Oceanum ipsum influat»). Dagli oceani, poi, il ciclo può ripartire e continuare all'infinito.

Questo "artificio matematico della natura” («naturae mathematica machinatio») è la vera causa della circolazione continua delle acque. Un gigantesco moto perpetuo, che - rimarca l'autore - s'accorda perfettamente con un passo biblico ineludibile per qualsiasi filosofo naturale europeo d'Età Moderna che intenda studiare i fiumi e le sorgenti: il primo capitolo del Qoelet («E Sacris Ecclesiastes Cap. I, num. 7, Omnia flumina intrant in mare, et mare non redundat. Ad locum, unde exeunt, flumina revertuntur, ut iterum fluant»). Per Bettini è proprio l'autorità di questo brano a confermare l'esistenza di un ciclo idrologico, il cui funzionamento può essere ricreato artificialmente ricorrendo a ingegnosi modelli meccanici (Fig. 1). Questi vengono descritti con cura nell'Apiaria, che è splendidamente e riccamente illustrato. Ma si tratta di modelli imprecisi, che hanno un valore esclusivamente esemplificativo: perché la scala ridotta della ricostruzione non permette al meccanismo di funzionare in piena autonomia. Occorre esercitare una forza esterna («impetum accepit a motrice potentiam»), surrogato dei giganteschi squilibri gravitazionali che generano il moto perpetuo delle acque nel pianeta. Bettini, del resto, non nasconde la sua sfiducia nei confronti della tecnica, che non è che una difettosa imitazione del perfetto creato. È soprattutto questa rivendicazione della sostanziale indipendenza della teoria interpretativa da qualsiasi verifica sperimentale ad allontanare il gesuita bolognese da altri autori - non pochi dei quali suoi confratelli - più propriamente e modernamente definibili come scienziati (Aricò 2002). Ma non dobbiamo dimenticare che è anche grazie al contributo di chi cercò e propose vie alternative e promiscue, per quanto perdenti alla luce della storia, se la strada (tuttora imperfetta) percorsa dalla scienza verso la comprensione della natura ha potuto evolversi nel corso dei secoli.

Acknowledgements: I wish to express my heartfelt thanks to the Linda Hall Library of Science, Engineering and Technology in Kansas City (MO, USA), and to the entire staff of the History of Science Center, for allowing me to conduct this research.

\section{BIBLIOGRAFIA}

Aricò D. (2002) - Riccioli nella cultura bolognese del suo tempo: il collegio, lo studio, le accademie. In: Borgato M.T. (ed.), Giambattista Riccioli e il merito scientifico dei gesuiti nell'età barocca. Olschki, Firenze.

Baldwin M. (2003) - Pious ambition. Natural philosophy and the Jesuit quest for the patronage of printed books in the Seventeenth Century. In: Feingold M. (ed.), Jesuit Science and the Republic of Letters. The MIT Press, Cambridge (MA)-London.

Bettini M. (1642) - Apiaria universae philosophiae mathematicae, in quibus paradoxa et nova pleraque machinamenta ad usus eximios traducta, et facillimis demonstrationibus confirmata. Tomus primus. Typis Io. Baptistae Ferronij, Bononiae.

Generali D. (1996) - La biblioteca gesuitica del Collegio Braidense di Milano. In: Beretta M., Mondella F., Monti M.T. (eds.), Per una storia critica della scienza. Cisalpino, Milano.

Luzzini F. (2013) - Il paradosso di Kircher (che paradosso non è). Acque Sotterranee 2013, 3: 65-66. DOI: 10.7343/AS-047-13-0074.

Maffioli C.S. (2010) - La via delle acque (1500-1700). Appropriazione delle arti e trasformazione delle matematiche. Olschki, Firenze, pp. 232-234.

Remmert V.R. (2006) - Picturing Jesuit Anti-Copernican consensus: astronomy and the biblical exegesis in the engraved title-page of Clavius's Opera mathematica (1612). In: O’ Malley J., Bailey G.A., Harris S.J., Kennedy T.F. (eds.), The Jesuits II: cultures, sciences, and the arts, 1540-1773. University of Toronto Press, TorontoBuffalo-London. 